Objective To control an outbreak of HIV among IVDUs Interventions A multidisciplinary outbreak control team was convened in 2008 and 2010 with representation from the Health Protection Unit, the Drug and Alcohol Action Team, Genitourinary Medicine, Infectious Diseases, and Virology to develop a control strategy. Traditional partner notification was supplemented by cluster testing among associates in housing projects and drop in centres used by IVDUs. Opportunistic screening of IVDUs attending health services was promoted. Laboratory investigations included phylogenetic analysis and the recent infection testing algorithm.

Outcomes A further 6 cases were identified in 2010: two through hospital screening and four through partner notification. Cluster testing did not yield any further cases among associates (0/29), but presented an opportunity to raise awareness of local HIV risk among IVDUs. Joint working between services was a feature of all interventions. All 8 cases 2008-2010 are the same strain, and 7/8 are linked to at least one other case. Despite extended surveillance at centres providing treatment for substance misuse, no further new cases have been identified since September 2010.

Conclusion An HIV outbreak among a hard-to-reach population has been successfully controlled by a prompt and close collaboration between services to deliver a range of complementary control strategies. We remain vigilant to the possibility of further cases since the source of one case has not been identified.

\section{P36 EVALUATION TO ASSESS PATIENTS' PERCEPTIONS OF RECEIVING THE RECENT INFECTION TESTING ALGORITHM [RITA] RESULT}

doi:10.1136/sextrans-2012-050601c.36

${ }^{1} \mathrm{~V}$ Gilbart, ${ }^{2} \mathrm{E}$ Anderson, ${ }^{*}{ }^{3} \mathrm{~N}$ Garrett, ${ }^{4} \mathrm{~S}$ Perera, ${ }^{5} \mathrm{M}$ Rayment, ${ }^{6} \mathrm{H}$ Williams, ${ }^{1} \mathrm{~J}$ H Tosswill, ${ }^{1} \mathrm{~V}$ Delpech. ${ }^{1}$ The Health Protection Agency; ${ }^{2}$ Royal Liverpool University Hospital, Liverpool, UK; ${ }^{3}$ Barts and the London NHS Trust, London, UK; ${ }^{4}$ Brighton and Sussex University Hospitals NHS Trust, Brighton, UK; ${ }^{5}$ Chelsea and Westminster NHS Foundation Trust, London, UK; ${ }^{6}$ Guy's and St Thomas' NHS Foundation Trust, London, UK

Background In the UK the primary objective of RITA has been to measure HIV incidence. The UK is the first country to routinely return results to patients and with over 7000 tests returned to clinicians so far, RITA is now seen as part of baseline HIV clinical care in participating centres.

Objectives To evaluate patients' experience of receiving a RITA result, including effects on identifying likely timing of infection, partner notification and relationships.

Methods Five HIV centres in England asked their patients to complete a questionnaire on their experience of receiving a "recently infected" RITA result soon after their HIV diagnosis.

Results Forty patients with newly diagnosed HIV and a "recent" RITA result completed the questionnaire ( $93 \% \mathrm{MSM}$ ). The majority $(30 / 40,75 \%)$ said that receiving the recent RITA result was helpful; 10 were unsure how helpful it was, and no participants found it unhelpful. 24 patients $(60 \%)$ reported RITA helped identify partners who may be at risk of HIV and 24 (62\%) felt it helped them to better understand how they may have been infected. Most (35, $88 \%$ ) either felt neutral or "not more upset" when they learnt that they were likely recently infected. Of those in a relationship 33/37 (89\%) reported RITA testing had not caused any relationship problems.

Conclusion The majority of patients receiving recent RITA results found this information helpful and no-one found it unhelpful. The result was felt to be useful in increasing an understanding of the likely timing of infection and for identifying "at risk" partners.

\section{P37 SEX, DRUGS, RISK TAKING AND STIS}

doi:10.1136/sextrans-2012-050601c.37

${ }^{1} \mathrm{~F}$ E Keane, ${ }^{1} \mathrm{E}$ Thomas, ${ }^{2} \mathrm{~A}$ Picton, ${ }^{1} \mathrm{~J}$ Palmer, ${ }^{1} \mathrm{~L}$ Haddon. ${ }^{*}$ Royal Cornwall Hospital, Cornwall, UK; ${ }^{2}$ Peninsula college of Medicine and Dentistry, Plymouth, UK

Background Hazardous alcohol consumption (HAC) and recreational drug (RD) use are associated with risky sexual behaviour but there is little hard evidence about actual sexually transmitted infection (STI) acquisition in these cohorts.

Aim To investigate the association between HAC, RD use, risky sexual behaviour and STI acquisition.

Methods Patients self-completed the Fast Alcohol Screening Test (FAST), a validated screening tool to identify HAC, and questions on $\mathrm{RD}$ use. Markers of sexual risk-taking and STI diagnoses were obtained from patient records. Three cohorts: those with/without HAC (ie FAST positive/ negative respectively), RD use ever/never and recent/no recent $\mathrm{RD}$ use were analysed. Statistical significance $(p<0.05)$ was calculated using Pearson's $\chi^{2}$ test.

Results Convenience sampling identified 221 new/re-book patients, 21 were excluded due to administrative errors. Respondents included 70 (35\%) men and 130 (65\%) women (age range, 15-69 years; median 25. $105(53.6 \%)$ and 91(46.4) identified as FAST positive and FAST negative, $85(43.6 \%)$ and $110(56.4 \%)$ reported RD use ever and never and $33(16.8 \%)$ and $163(83.2 \%)$ reported $\mathrm{RD}$ use and no $\mathrm{RD}$ use in the last $1 / 12$ respectively. $67 / 102$ FAST positives reported a new sexual partner in the previous $3 / 12$ compared to 29/88 FAST negatives $(p<0.001)$. Recent RD users were also more likely to report partner change $(23 / 32$ compared to those with no recent $R D$ $(75 / 159, \mathrm{p}=0.011)$. There was an increase in STI diagnoses in FAST positive compared to FAST negative patients (34/105 and 17/91 $\mathrm{p}=0.029$ ), no difference was observed in the other cohorts.

Discussion HAC is very common among our patients and appears to be linked to increased STI acquisition. Recent $\mathrm{RD}$ use is not uncommon. Our findings support the recommendations of the recent Alcohol and Sexual Health Working Party that identification of hazardous drinkers and brief interventions may improve sexual health outcomes.

\section{P38 QUANTIFYING THE PUBLIC HEALTH VALUE OF PROVIDER-LED PARTNER NOTIFICATION USING AN EVIDENCE-BASED ALGORITHM WITH ROUTINELY- COLLECTED DATA}

doi:10.1136/sextrans-2012-050601c.38

${ }^{1} \mathrm{C}$ Mercer, ${ }^{*}{ }^{2} \mathrm{G}$ Bell, ${ }^{3} \mathrm{~N}$ Low, ${ }^{4} \mathrm{C}$ S Estcourt, ${ }^{5} \mathrm{G}$ Brook, ${ }^{6} \mathrm{~J}$ A Cassell. ${ }^{1}$ University College London, London, UK; ${ }^{2}$ Royal Hallamshire Hospital, Sheffield, UK; ${ }^{3}$ University of Bern, Bern, Switzerland; ${ }^{4}$ Queen Mary, University of London, London, UK; ${ }^{5}$ Central Middlesex Hospital, London, UK; ${ }^{6}$ Brighton and Sussex Medical School, Brighton, UK

Objectives To assess (i) the public health benefit of provider-led partner notification (PN) and (ii) the importance of taking into account the number and types of partners when assessing the costbenefit of provider-led PN.

Method We used data on 346 partners reported by 220 chlamydia index patients ( $\mathrm{n}=127$ men) seen by a PN professional at one GUM clinic, April-June 2011. We used a published, evidence-based algorithm to quantify PN impact, including the measure the "Number Needed to Treat to Interrupt Transmission" (NNTIT), defined as the number of partners who need to be treated to interrupt 1 onward (secondary) transmission. A lower NNTIT indicates less PN effort is required to prevent onward transmission.

Results Men reported 216/346 partners and women 130/346. Men more often described partners as casual ( $56 \%$ vs $28 \%$ ), while women more often reported regular ( $40 \%$ vs $30 \%$ ) and ex-partners (23\% vs 
$12 \%$ ). For traceable partners (73\% of men's; $88 \%$ of women's), 9/157 men's partners and 16/114 women's partners required provider-led PN. Almost all were casual or ex-partners (8/9 men's; 13/16 women's). NNTITs were lowest for ex-partners: 0.6 and 0.45 for men and women, respectively, followed by casual partners: 0.8 and 0.6 , then regular partners: 1.80 and 0.9 .

Discussion Provider-led PN is required more often with casual and ex-partners than with regular partners. This may have greater cost implications for services, but, as casual and ex-partners are likely to have greater numbers of partners themselves, the potential for preventing onward transmission is greater, yielding greater public health benefit. Services should collect data from their index patients on the number and type(s) of partners, as well as the PN method(s) required (patient vs provider referral). Together with cost data, these data were key for demonstrating the cost-benefit of provider-led PN.

\section{P39 THE ACCEPTABILITY AND USE OF SMS TEXT MESSAGING FOR PROVIDER REFERRAL PARTNER NOTIFICATION (PRPN)}

doi:10.1136/sextrans-2012-050601c.39

V Gilbart, ${ }^{*}$ K Town, C M Lowndes. The Health Protection Agency

Background Partner notification is important for STI control. With developments in technology notifying partners is now easier. The HPA was keen to understand the acceptability and use of SMS texting for PRPN for STIs.

Methods A questionnaire distributed to GUM colleagues, cascaded by the Society of Sexual Health Advisers and posted on their website.

Results 65 sites responded. Most (61,93\%) use telephone for 1st attempt for PRPN with texting as preferred second choice $(19,29 \%)$. For 2nd attempt, telephone remained preferred method (51, 79\%) but texting increased with $32(49.2 \%)$ choosing this method; letter use also increased. Majority of sites used texting for PRPN (56, $(86 \%)$; frequency varied-over half using it up to $50 \%$ of the time. $29(45 \%)$ had texting guidelines and 31 (55\%) used texting templates. Messages varied; 33 (59\%) requesting partner make contact, 9 (16\%) naming the infection. Six (11\%) had contact with Information Governance Board about texting; no negative outcomes reported. Two partners complained, not about receiving a text but about being denied the identity of the index patient. 9 (14\%) sites never used texting.

Conclusion Telephone contact was the preferred option for 1st and 2nd attempt for PRPN. Texting was the 2 nd most used method and was seen as acceptable within the clinic and also with Information Governance Board. Frequency varied considerably and message content differed but even if used infrequently, was seen as important if other methods failed. Reassuringly no complaints about texting were received from partners. A small number of sites never use texting.

\section{P40 LOSING THE BATTLE? TIME TO FOCUS THE RISK DISCUSSION WITH HIV-POSITIVE MSM}

doi:10.1136/sextrans-2012-050601c.40

S Perera, ${ }^{*}$ S McCormack, N Nwokolo. Chelsea and Westminster Hospital NHS Foundation Trust, London, UK

Background BHIVA 2008 Guidelines advocate development of prevention messages promoting sexual health of HIV-positive individuals to minimise onward transmission and acquisition of infections prevalent in such individuals, for example HCV and LGV.
Objectives To assess the extent of unprotected anal intercourse (UAI) in an HIV-positive population attending a London sexual health clinic. To assess transmission and acquisition risks. To describe the nature of sexual health discussions with health care providers.

Methods 50 randomly selected notes of HIV-positive patients were reviewed. Data were collected on demographics, ART, viral load (VL), sexual partners and topics discussed.

Results 66\% were from the UK and 74\% were $>30.47$ (94\%) were MSM/Bisexual; 2 (4\%) heterosexual females; for 1 (2\%) male IVDU, orientation, partners and discussion were not documented. In 8/47 MSM it was unclear if AI had occurred; 1/47 reported AI but condom use was not documented. In 38 sufficiently complete records, all reported AI: 7 (18\%) protected AI only, 12 (32\%) UAI with positive partners only; $19(50 \%)$ reported UAI with positive and negative partners, although $9 / 19$ (47\%) had VL $<500.7 / 10$ MSM reporting UAI who had VL $>500$ used recreational drugs although only one reported doing so during sex. Discussion was not documented in 17 (34\%) notes, and in 12 focus was only on transmission. 19 topics were discussed with no consistency but could be classified as transmission or acquisition related. Transmission risks were discussed in 15 (79\%) of the 19 reporting UAI with negative partners; acquisition risks were discussed in 17 (55\%) of 31 at risk.

Conclusions Although UAI was reported by the majority of MSM only 10 reported a clinically relevant risk of transmitting HIV, but the majority were at risk of acquiring HCV/LGV. The diversity in topics noted as discussed suggests that simpler, more focused messages of risk reduction would be helpful to sexual health service users and providers.

\section{P41 WOMEN'S HEALTH STUDY OF MYCOPLASMA GENITALIUM: A FEASIBILITY STUDY}

doi:10.1136/sextrans-2012-050601c.41

${ }^{1} \mathrm{~S}$ Dave, ${ }^{*} \mathrm{~S}$ R Kerry, ${ }^{2} \mathrm{P}$ Oakeshott, ${ }^{3} \mathrm{~F}$ McGregor, ${ }^{3} \mathrm{E}$ Cannon, ${ }^{1} \mathrm{~J} \mathrm{M}$ Stephenson. ${ }^{1}$ University College London, London, UK; ${ }^{2} S$ t. George's University of London, London, UK; ${ }^{3}$ CNWL Camden Provider Services, London, UK

Background The role of Mycoplasma genitalium ( $~ 3 \%$ prevalence in women) in PID is unclear. An opportunity exists to study its natural history since there are no UK treatment guidelines. We conducted a feasibility study.

Objectives In women attending GUM clinics to determine: (1) recruitment and follow-up rates after 3 and 6 months, (2) feasibility of obtaining NHS numbers, (3) facilitators and barriers to follow-up. Methods Women aged 16-24 years recruited from two GUM clinics completed a questionnaire and provided two self-taken vaginal samples. One sample was tested for chlamydia. We are testing for $M$ genitalium and conducting 3-month follow-up.

Results Recruitment rate was 67\% (199/296). Non-responders were similar in age to responders (20.6 SD 2.5 vs 20.7 SD 2.5 years) but more likely to come from ethnic minority groups: $56 \%(37 / 66)$ vs $36 \%(71 / 195), p<0.05$. Among responders, mean sexual debut age was 16.3 years (range $12-23 n=198) ; 59 \%(116 / 195)$ reported $\geq 2$ sexual partners and $17 \%(31 / 186)$ consistently used condoms in the preceding 12 months. $8.2 \%(16 / 197)$ had chlamydia and $0.5 \%$ (1/ 197) gonorrhoea on testing. At recruitment 196 participants consented to NHS numbers being obtained and used to access medical records; 197 participants agreed to 3-month follow-up. We have been able to contact $\sim 50 \%$ of participants for follow-up.

Discussion Most women consented to NHS number and medical record access. This is important for investigating long-term sequelae of STIs. Fewer women took part in follow-up than agreed at recruitment. It is important to understand the reasons for this. 